Supplementary text:

\title{
Molecular modelling
}

PSI-Blast (http://www.ncbi.nlm.nih.gov/BLAST/) [1] was used to identify the lipocalins most similar to AGP. Human AGP-1 protein sequence from UniProt (http://www.uniprot.org/) [2], accession no. P02763, was used as the query against the $n r$ database as of March $8^{\text {th }}$, 2006. The major horse allergen Equ c 1 was identified as the closest non-AGP lipocalin in the second PSI-Blast iteration. The structure of the Equ c 1 allergen is available in PDB (http://www.pdb.org/) [3] as 1EW3 [4], and it was selected as the major template for modelling. Other structures for reference and for multiple sequence alignment were identified from further PSI-Blast iterations and from the Dali database of structural similarity (http://ekhidna.biocenter.helsinki.fi:9801/dali/start, update of March 2005) [5]. Coordinates for 1EW3, 1GM6, 2A2U, 1BJ7, 1E5P, and 1DF3 were retrieved from PDB. Twelve AGP protein sequences from nine species (including two human and three mouse isoforms) were retrieved from UniProt and Ensembl (http://www.ensembl.org/) [6]. A multiple sequence alignment of these sequences and the six template proteins was constructed with TCoffee (http://www.igs.cnrsmrs.fr/Tcoffee/tcoffee_cgi/index.cgi) [7]. The alignment of the PDB proteins was found to be consistent with structure-based pairwise alignments against 1EW3 (from the Dali database). Two gaps were observed within the regions corresponding to lipocalin beta strands $\mathrm{B}$ and $\mathrm{C}$. The gaps were moved to $\mathrm{AB}$ and $\mathrm{BC}$ loops, respectively, by manual editing. The final alignment and further details of the sequences are available in Supplementary Materials. Our alignment was given as input to the Alignment interface of the SwissModel server (http://swissmodel.expasy.org/) [8] to receive the initial model of human AGP-1 built with 1EW3 as template, covering residues 11-170 of AGP-1. Rotamers of several side chains were adjusted manually and the loop 91 to 95 which blocked the ligand binding pocket was rebuilt in Discovery Studio 1.5. (Accelrys, San Diego, CA). Hydrogens were added and the structure was energy minimized, relaxing first hydrogens, then side chains, then backbone of the new 
loops but leaving the backbone of secondary structures fixed. The model shows reasonable bond lengths and angles, and only 11/160 residues (6.9\%) with poor phi/psi values in the Ramachandran plot. The previous AGP models $[9,10]$ are mutually quite different in their alignment, with only 74 residues in matching positions, whereas each previous model matches with 109/160 (partly different) residues in our model, and only 17 residues in our model are in positions not found in either model. The available templates do not allow accurate modelling of the first 10 or the last 13 to 18 residues of any known AGPs. According to our model, the terminal sequences would be located far from the binding site and their direct participation in ligand binding is unlikely.

To add oligosaccharide structures to this glycoprotein, the model was stripped of hydrogens and uploaded to the GlyProt server (http://www.glycosciences.de/modeling/glyprot/php/main.php) [11]. We selected fully sialylated, non-fucosylated complex-type glycans from the associated Glycom-DB of $\mathrm{N}$-linked glycan structures for the five glycosylation sites according to known preferences of each site. A trisialylated triantennary glycan (entry 9085) was inserted at N15 and N45, a bisialylated biantennary standard glycan at N38, and a tetrasialylated tetraantennary glycan (entry 9116) at N75 and N85. The resulting in silico glycosylated model was returned to Discovery Studio for addition and relaxation of hydrogens and manual adjustments of glycan chains to leave the binding site free and to eliminate bad contacts. The structural quality of the final model and various intermediates were checked with WhatCheck (http://swift.cmbi.kun.nl/WIWWWI/modcheck.html). Final molecular images and animations were prepared with PyMol (DeLano Scientific LLC, South San Francisco, CA).

\section{Docking of LPC into the model}

Crystal structure of a deoxy-LPC (3-dodecanoyl-propanediol-1-phosphorylcholine) was obtained from the Cambridge Structural Database (Cambridge Crystallographic Data Centre, Cambridge, UK; entry 
LYPCHL) [12]. The structure was modified in Discovery Studio: 2-OH was added in the glycerol moiety and the dodecanoyl chain was replaced by arachidonic acid (sn1-20:4 $:^{\Delta, 8,11,14}$, all-cis) in an extended conformation. All $\mathrm{COO}^{-}$and $\mathrm{NH}_{3}{ }^{+}$groups in the AGP-1 model and the phosphate in the ligand were made explicitely charged by inclusion or omission of appropriate hydrogens. GOLD software (Cambridge Crystallographic Data Centre, Cambridge, UK) was used for flexible docking of the ligand into the protein. The centre for search was set at the CG atom of Glu-96 on one side of the pocket, radius as $13 \AA$, and the genetic algorithm search was run to produce 24 dockings for the ligand. 


\section{References}

1 Altschul, S. F., Madden, T. L., Schäffer, A. A., Zhang, J., Zhang, Z., Miller, W., and Lipman, D. J. (1997) Gapped BLAST and PSI-BLAST: a new generation of protein database search programs, Nucleic Acids Res. 25, 3389-3402.

2 Bairoch, A., Apweiler, R., Wu, C.H., Barker, W.C., Boeckmann, B., Ferro, S., Gasteiger, E., Huang, H., Lopez, R., Magrane, M., Martin, M.J., Natale, D.A., O'Donovan, C., Redaschi, N., and Yeh, L.S. (2005) The Universal Protein Resource (UniProt) Nucleic Acids Res. 33, D154-159.

3 Berman, H. M., Westbrook, J., Feng, Z., Gilliland, G., Bhat, T. N., Weissig, H., Shindyalov, I. N., and Bourne, P. E. (2000) The Protein Data Bank, Nucleic Acids Res. 28, 235-242.

4 Lascombe, M.B., Gregoire, C., Poncet, P., Tavares, G.A., Rosinski-Chupin, I., Rabillon, J., Goubran-Botros, H., Mazie, J.C., David, B., and Alzari, P.M. (2000) Crystal structure of the allergen Equ c 1. A dimeric lipocalin with restricted IgE-reactive epitopes. J. Biol. Chem. 275, 21572-21577.

5 Holm, L., and Sander, C. (1996) Mapping the protein universe. Science 273, 595-603.

6 Hubbard, T. Andrews, D., Caccamo, M., Cameron, G., Chen, Y., Clamp, M., Clarke, L., Coates, G., Cox, T., Cunningham, F., Curwen, V., Cutts, T., Down, T., Durbin, R., Fernandez-Suarez, X. M., Gilbert, J., Hammond, M., Herrero, J., Hotz, H., Howe, K., Iyer, V., Jekosch, K., Kahari, A., Kasprzyk, A.. Keefe, D., Keenan, S., Kokocinsci, F., London, D., Longden, I., McVicker, G., Melsopp, C., Meidl, P., Potter, S., Proctor, G., Rae, M., Rios, D., Schuster, M., Searle, S., Severin, J., Slater, G., Smedley, D., Smith, J., Pooner, W., Stabenau, A., Stalker, J., Storey, R., Trevanion, S., Ureta-Vidal, A., Vogel, J., White, S., Woodwark, C., and Birney, E. (2005) Nucleic Acids Res. 33, D447-D453.

7 Poirot, O., Suhre, K., Abergel, C., O'Toole, E., and Notredame, C. (2004) 3DCoffee: a web server for mixing Sequences and Structures into multiple sequence alignments. Nucleic Acids Res. 32, W37-40. 
8 Schwede, T., Kopp, J., Guex, N., and Peitsch, M.C. (2003) SWISS-MODEL: an automated protein homology-modeling server. Nucleic Acids Res. 31, 3381-3385.

9 Kopecky, V. Jr., Ettrich, R., Hofbauerova, K., and Baumruk, V. (2003) Structure of human alpha1acid glycoprotein and its high-affinity binding site. Biochem. Biophys. Res. Commun. 300, 41-46.

10 Rojo-Dominguez, A., and Hernandez-Arana, A (1993) Three-dimensional modeling of the protein moiety of human a1-acid glycoprotein, a lipocali-family member. Protein Seq. Data Analys. 5, 349355.

11 Bohne-Lang, A., and von der Lieth, C. W. (2005) GlyProt: in silico glycosylation of proteins. Nucleic Acids Res. 33, W214-219.

12 Hauser, H., Pascher, and I., Sundell, S. (1980) Conformation of phospholipids. Crystal structure of a lysophosphatidylcholine analogue. J. Mol. Biol. 137, 249. 


\section{Figure legends}

1 The alignment of 12 alpha-1-glycoprotein sequences from nine species and six structurally known lipocalin sequences. Shading according to similarity in each column (amino acid position). For sources and details of the sequences, see sequences_AGP.pdf.

2 The protein sequences used in the multiple sequence alignment, in Fasta format. The first six sequences are from PBB, and their accession codes are the first four characters of the sequence names (after the ">" character). The next eight are from UniProt and the last four from Ensembl, with their respective codes after the seqence name. Presumed signal sequences (first 18 residues) have been omitted from all UniProt and Ensembl sequences. 


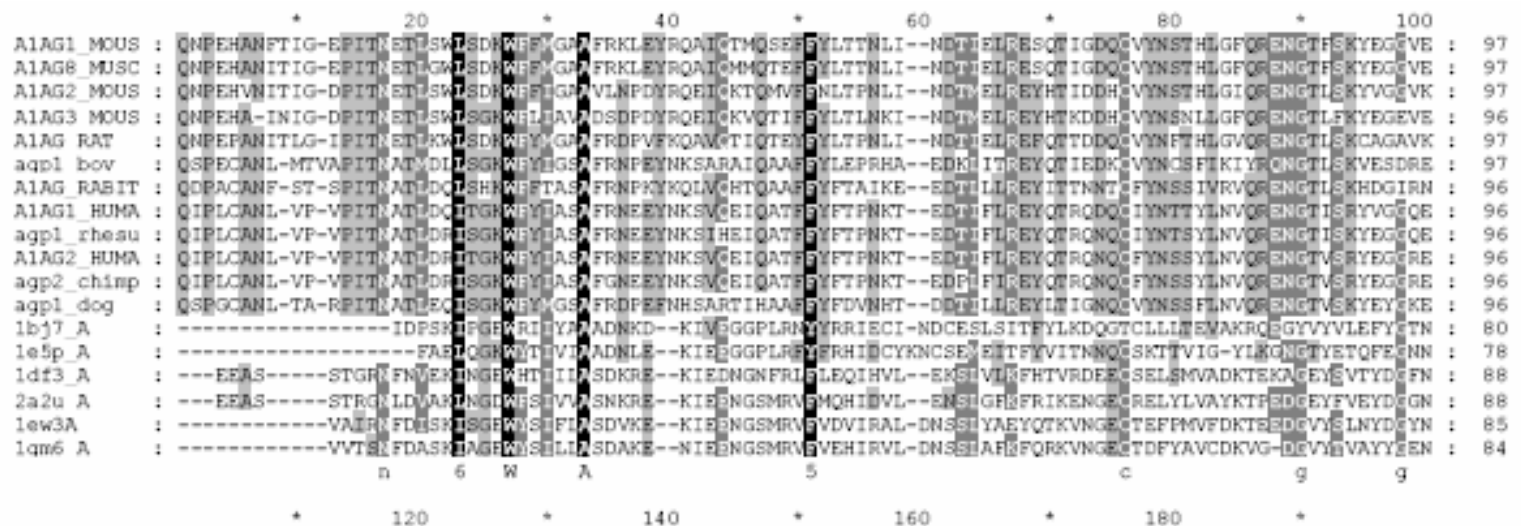

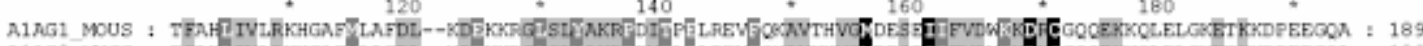

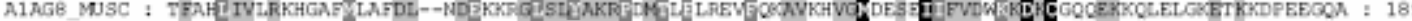

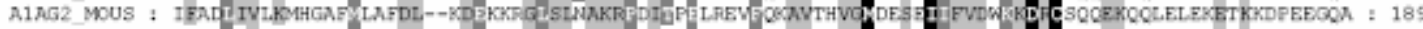

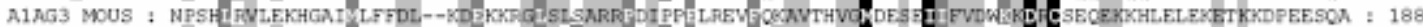

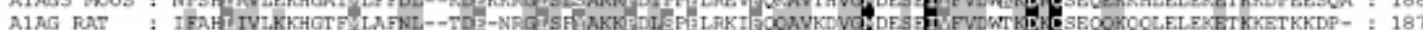

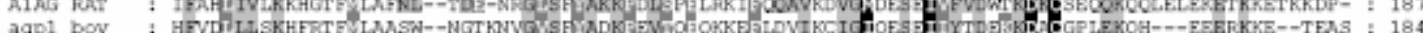

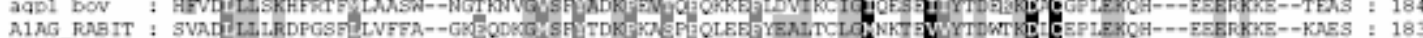

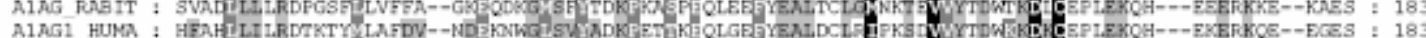

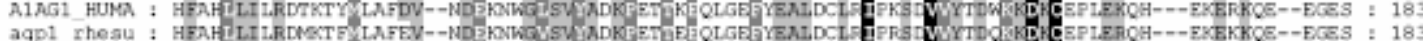

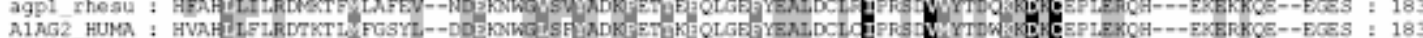

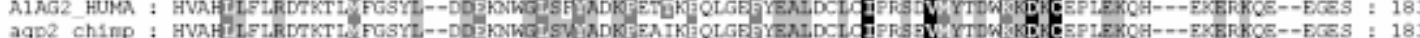
agp2 ChInp : AVRHLLFLRDTKTL FGSY--DDEKNh LSW

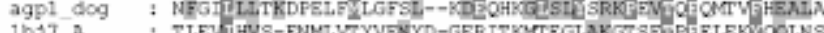

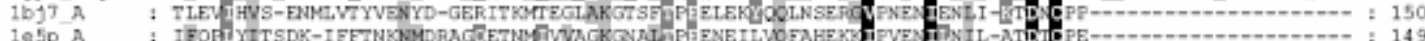

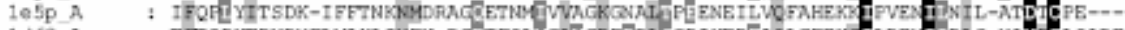

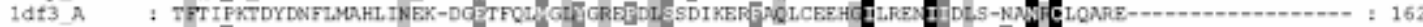

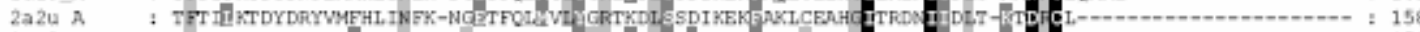

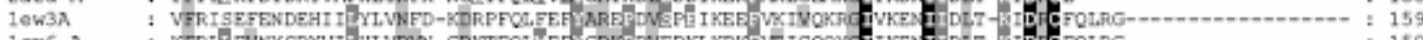

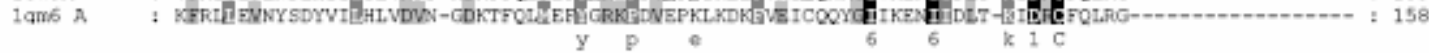

\section{Supplementary figure 1}


$>1$ ew $3 \mathrm{~A}$

VAIRNFDISKISGEWYSIFLASDVKEKIEENGSMRVFVDVIRALDNSSLYAEYQTKVNGECTEFPMVFDK TEEDGVYSLNYDGYNVFRISEFENDEHI ILYLVNFDKDRPFQLFEFYAREPDVSPEIKEEFVKIVQKRGI

VKENIIDLTKIDRCFQLRG

$>1 \mathrm{gm} 6 \mathrm{~A}$

VVTSNFDDASKIAGEWYSILLASDAKENTEENGSMRVEVEHIRVLDNSSLAFKF

QRKVNGECTDFYAVCDKVGDGVYTVAYYGENKFRLLEVNYSDYVILHLVDVNGDKTFQLM

EFYGRKPDVEPKLKDKFVEICQQYGIIKENIIDLTKIDRCFQLRG

$>2 a 2 u_{A}$ A

EEASSTRGNLDVAKLNGDWFSIVVASNKREKIEENGSMRVF

MQHIDVLENSLGFKFRIKENGECRELYLVAYKTPEDGEYFVEYDGGNTFTILKTDYDRYV

MFHLINFKNGETFQLMVLYGRTKDLSSDIKEKFAKLCEAHGITRDNIIDLTKTDRCI

$>1 \mathrm{bj} 7 \mathrm{~A}$

IDPSKI PGEWRI IYAAADNKDKIVEGGPLRNYYRRIECINDCESLSITFYLKDQ

GTCLLLTEVAKRQEGYVYVLEFYGTNTLEVIHVSENMLVTYVENYDGERITKMTEGLAKG

TSFTPEELEKYQQLNSERGVPNENIENLIKTDNCPP

$>1 e 5 p_{-}$A

FAELQGKKWYTIVIAADNLEKIEEGGPLRFYFRHIDCYKNCSEMEITFYVITNNQCSKT

TVIGYLKGNGTYETQFEGNNIFQPLYITSDKIFFTNKNMDRAGQETMMIVVAGKGNALTP EENE ILVQFAHEKKIPVENILNILATDTCPE

$>1$ df 3 A

EEASST TRNFNVEKINGEWHTI ILASDKREKIEDNGNFRLFLEQIHVLEKSLVLKFHTVR DEECSELSMVADKTEKAGEYSVTYDGFNTFTIPKTDYDNFLMAHLINEKDGETFQLMGLY GREPDLSSDIKERFAQLCEEHGILRENI IDLSNANRCLQARE

$>$ A1AG1 HUMAN P02763

QIPLCANNLVPVPITNATLDQITGKWFYIASAFRNEEYNKSVQ

QIOATFFYFTPNKTEDTIFLREYQTRQDQCIYNTTYLNVORENGTISRYVGGQEHFAHLL ILRDTKTYMLAF DVNDEKNWGLSVYADKPETTKEQLGEFYEALDCLRIPKSDVVYTDWKK DKCEPLEKQHEKERKQEEGE

$>$ A1AG2_HUMAN P19652

QIPLCANILVPPITNATLDRITGKWFYIASAFRNEEYNKSVQ

EIQATFFYFTPNKTEDTIFLREYQTRQNQCFYNSSYLNVQRENGTVSRYEGGREHVAHLL FLRDTKTLMFGSYLDDEKNWGLSFYADKPETTKEQLGEFYEALDCLCIPRSDVMYTDWKK

DKCEPLEKQHEKERKQEEGES

QDPACĀNF STSPITNATLDQLSHKWFFTASAFRNPKYKQLVQ

HTQA.FFYFTAIKEEDTLLLREYITTNNTCFYNSSIVRVQRENGTLSKHDGIRNSVADLL LLRDPGSFLLVFFAGKEQDKGMSFYT DKPRAS PEQLEEFYEALTCLGMNKTEVVYTDWTK DLCEPLEKQHEEERKKEKAES

A1AG8 MUSCR P21352

QNPEHAÑNITIGEPITNETLGWLSDKWFFMGAAFRKLEYRQAI

QMMOTEFFYLTTNL INDTIELRESQT IGDQCVYNSTHLGFQRENGTF SKYEGGVETFAHL IVLRKHGAFMLAFDLNDEKKRGLSLYAKRPDMTLELREVFQKAVKHVGMDESEI IFVDWK KDKCGQQEKKQLELGKETKKDPEEGQA

$>A 1 A G 1$ MOUSE Q60590

QNPEHAN $\bar{N}$ TIGEPITNETLSWLSDKWFFMGARARKLEYRQAI

OTMOSEFFYLTTNL INDTIELRESOTIGDOCVYNSTHLGFORENGTFSKYEGGVETFAHL IVLRKHGAFMLAFDLKDEKKRGLSLYAKRPDITPELREVFOKAVTHVGMDESEI I FVDWK KDRCGQQEKKQLELGKETKKDPEEGQA

A.1AG2 MOUSE P07361

QNPEHVNIITIGDPITNETLSWLSDKWFFIGAAVLNPDYRQEI

QKTQMVFFNLTPNLINDTMELREYHT IDDHCVYNSTHLGIQRENGTLSKYVGGVKIFADL IVLKMHGAFMLAFDLKDEKKRGLSLNAKRPDITPELREVFQKAVTHVGMDESEIIFVDWK KDRCSQQEKQQLELEKETKKDPEEGQA

A.1AG3 MOUSE Q63805

QNPEHA INNIGDPITNETLSWLSGKWFLIAVADSDPDYRQEIO

KVOTIFFYLTLNKINDTMELREYHTKDDHCVYNSNLLGFORENGTLFKYEGEVENPSHLR VLEKHGAIML FFDLKDEKKRGLSL SARRPDIPPELREVFOKAVTHVGMDESEI IFVDWKK DRCSEQEKKHLELEKETKKDPEESQA

DRCSEQEKKHLELEKE
$>$ A1AG RAT P02764

PA1AG RAT P02764

QTIQTEYFYLTPNL INDTIELREFQTTDDQCVYNFTHLGVQRENGTLSKCAGAVKIFAHL IVLKKHGTFMLAFNLTDENRGLSFYAKKPDLSPELRKIFQQAVKDVGMDESEIVFVDWTK DKCSEQQKQQLELEKETKKETKKDP

agp1 dog ENSCAFP00000004937

QSPGCANLTARPITNATLEOISGKWFYMGSAFRDPEFNHSAR

TIHA.AFFYFDVNHTDDTILLREYLTIGNOCVYNSSFLNVORENGTVSKYEYGKENFGILL LTKDPELFML GFSLKDEOHKGLSLYSRKPEVTOEOMTVFHEALACMDLHKSEIAYTDEKK LTKDPELFMLGF SLKDE

agp 1 bov ENSBTAP0000002299

QSPECĀNLMTVAPITNATMDLLSGKWFY I GSAFRNPEYNKSA

RAIQAAFFYLEPRHAEDKLITREYQT IEDKCVYNCSFIKIYRQNGTLSKVESDREHFVDL LLSKHFRTFMLAASWNGTKNVGVSFYADKPEVTQEQKKEFLDVIKCIGIQESEIIYTDEK KDACGPLEKQHEEERKKETEAS

>agp1 rhesus ENSMMUP00000010511

QIPLCĀNLVPVPITNATLDRISGKWFYIASAFRNEEYNKSIH

EIQATFFYFTPNKTEDTIFLREYQTRQNQCIYNT SYLNVQRENGTISKYEGGQEHFAHLL ILRDMKTFMLAFEVNDEKNWGVSVYADKPETTEEQLGEFYEALDCLRIPRS DVVYTDQKK DKCEPLEROHEKEKKOEEGES

OCD 2 chimp ENSPTRP00000036363

QIPLCANNLVPVITNATLDRISGKWFYIASAFGNEEYNKSVQ

EIQATFFYFTPNKTEDPLFIREYQTRQNQCFYNSSYLNVQRENGTVSRYEGGREHVAHLI FLRDTKTLMFGSYLDDEKNWGLSVYADKPEAIKEQLGEFYEALDCLCIPRSEVMYTDWKK DKCEPLEKQHEKERKQEEGES

\section{Supplementary figure 2}

